Ann. Abeille, I967, 10 (I), 29-37.

\title{
ÜBER DAS AUFTRETEN VON PYLORUSSCHORFEN BEI GESUNDEN UND NOSEMAKRANKEN ARBEITERINNEN DER HONIGBIENE UNTER VERSCHIEDENEN EXPERIMENTELLEN BEDINGUNGEN
}

\author{
R. BÄIIRMANN
}

Institut für Spesielle Zoologie und Enlomologie, 69-Jena, Allemagne (D. D. R.)

Bei 24 Tage alten gesunden und nosemakranken Arbeiterinnen der Honigbiene Apis mellifica wurde das Auftreten und die Stärke der Pylorusschorfe morphologisch untersucht. Die Bienen waren während der 24 Tage unter verschiedenen experimentellen Bedingungen gehalten worden : mit Pollenzufütterung, ohne Zufütterung von Pollen, in Gesellschaft von älteren Bienen und unter Verfütterung von Darmsaft älterer Bienen. Es zeigten sich Unterschiede in der Ausbildung der Pylorusschorfe bei den einzelnen Versuchsgruppen. Die Unterschiede werden hervorgehoben und diskutiert.

\section{EINLEITENDE FRAGESTELLUNG}

Wie bei den Arthropoden allgemein kommen auch bei den Insekten Flagellaten im Enddarm verhältnismässig häufig vor und können an der Darmwand sogenannte " Polster » bilden (NÖLLER, I93I). LOTMAR (I946) fand im Pylorusbereich von Honigbienen Flagellaten, die frei im Lumen, aber auch sessil vorhanden sind. Das Vorkommen der sessilen Formen ist jedoch auf einen engen Raum begrenzt. Schon LOTMAR (I946) macht auf Schorfbildungen an der Darmwand aufmerksam, die ursächlich auf die sessilen Flagellaten zurückgeführt werden; HischIER (I962) vertritt die Ansicht, dass die Pylorusflagellaten anscheinend regelmässig an ihrer 
Ansatzstelle im Enddarm Schorfe bilden. Auch Gravarisi (I950) fand diese Flagellatenschorfe. Nach den genannten Autoren befinden sich die Schorfbildungen auf der Dorsalseite des Pylorus. Während nach Giavarins kein Zusammenhang zwischen dem Auftreten der Flagellaten und der Zusammensetzung der Nahrung zu bestehen scheint, lässt LoTMAR diese Frage offen. Nach FYG (1954) ist es jedoch möglich, dass die Entwicklung der Pylorusflagellaten weitgehend von der jeweiligen Nahrung der Wirtstiere und auch von der Temperatur abhängt (Abb. I et 2).

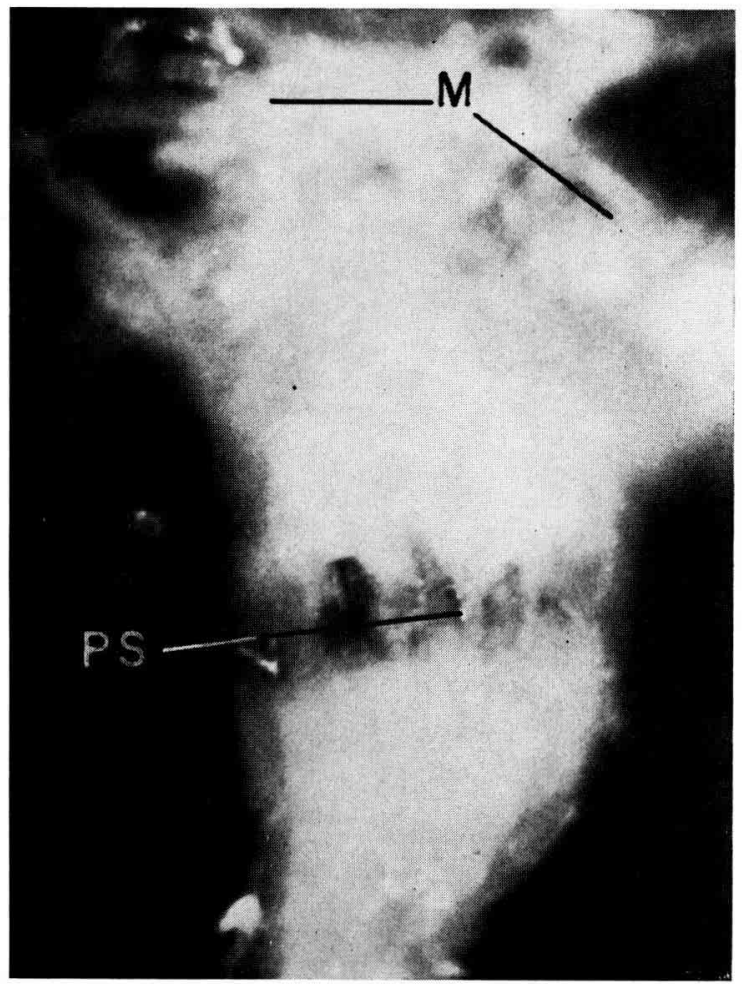

Abb. 1. - Pylorusabschnill des Dimnularmes einer Arbilsbiene (Dorsulansichl),

der sich durch einen ring/ärmigen Besalz a'on I'ylorusschorjen unlerschiedlicher Slärke ausceichnet

Die Pylorussichorfe (PS) sind durch die helle Darmwand gut za sehen.

1], Ialpichische Gefässe ; Vergrösseruner : 60 fach

FIG. I. - Section lu fylore an nivean de linteslin grile d'une onorière (rute dorsale), se distingatanl par la présence d'escarres en forme 't'anneal, d'intensilé distincte.

Ies escarres du pylore (PS) sont bien visibles à travers la paroi claire de l'intestin II, tubes re Malpighi. Grossissement : $\sim 60$ fois

In mehrjährigen Untersuchungen der Flagellatenschorfe bei Winterbienen konnte festgestellt werden, dass bei verschiedenen Völkern, die unter annähernd gleichen Bedingungen gehalten worden waren, die Häufigkeit des Auftretens von Pylorusflagellaten beträchtlichen Schwankungen unterliegen kann (BÄHRMANN, noch unveröffentlicht). So ergibt sich die Frage, welche Faktoren wohl für das Zustandekommen der Flagellatenpolster und damit für die Ausbildung der Pylorus- 
schorfe verantwortlich sein mögen. In den vorliegenden Untersuchungen soll geprift werden, welchen Einfluss die Pollennahrung und der Kontakt zwischen älteren und jüngeren Arbeitsbienen auf die Bildung der Pylorusschorfe ausüben.

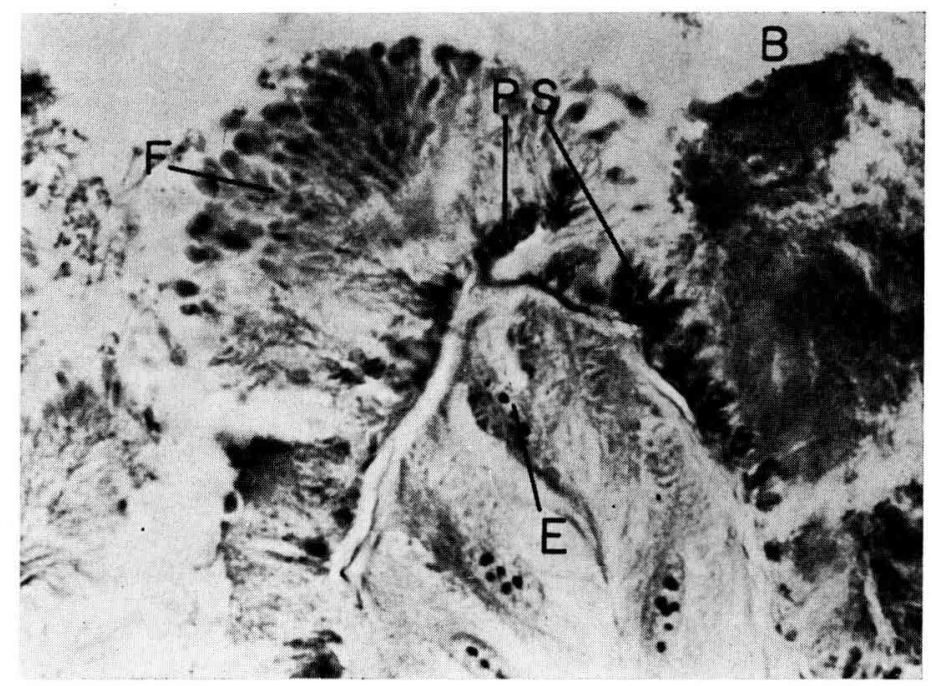

ABB. 2. - Querschnitt durch den Pylonusabschnitt des Dünndarmes einer Arbeilsbiene

Unter dem Flagellatenbesatz (F) hat dic Schorfbildung (P'S) eingesetzt. 13, Bakterien; E, Jipithelfalte ; Färbung : Mallory-IIeidenhain; Schnittdicke : $7 \mu m$; Vergrösserung : Objektiv $60 \times$, l'rojektiv $4 \times$.

Fic. 2. - Coupe Iransversule à travers le pylore de l'intestin grêle d'une ouvrière

Les escarres (PS) sont apparues au milicu du peuplement des flagellés (li). B, Bactéries ; L, Repli épithélial. Coloration : Mallory-Ileidenhain. Evaisseur de la coupe $7 \mathrm{~km}$. (rossissement : Olbjectif $60 \mathrm{x}$, Oculaire $+x$

\section{VERSUC'HSME'THODIK}

Zwischen dem i6. und I8. V. 1966 wurden mehrere Versuchsküstchen, mit den Aussenmassen $4,5 \mathrm{~cm} \times 10,2 \mathrm{~cm} \times 12 \mathrm{~cm}$, mit je I 20 frisch geschlüpften $A$ rbeitsbienen besiedelt. Die Bienen kamen in Wärmeschrank be $3^{\circ} \mathrm{C}$ zum Schlüpfen. In den darauf folgenden 24 Tagen betrug die Kästchentemperatur $30^{\circ} \mathrm{C}$. Diese Temperatur entspricht nach Mavrizio (1946) den Bedürfnissen gefangen gehaltener Bienen am besten, was sich in der relativ geringen Sterblichkeit auswirkt. In zwei Kästchen wurde den Bienen neben Futterteig (das in der Inkerei gebräuchliche Honig-StaubzuckerGemisch) nur Wasser in zwei Tränkröhrchen beigegeben, in zwei weiteren Kästchen neben dem Futterteig und dem Wasser auch noch ab I. Tag frischer, einer Wabe entnommener Pollen gefüttert. $\mathrm{Zu}$ je einer der beiden Versuchsgruppen gab ich 40 Stockbienen aus dem Baurahmen. Dabei handelt es sich um Bienen, die ihrer 'Tätigkeit entsprechend mindestens eine Woche alt gewesen sind. In drei weiteren Kästchenversuchen wurde den je I 20 frisch geschlüpften Bienen I) Pollen, Futterteig, Wasser und Darmsaft von Io zerriebenen Biendärmen (Einddärmen) gegeben. Es gelangten dabei nur solche Därme zur Verwendung, die nach vorheriger Kontrolle als nosemafrei befunden worden waren. 2) Verfüttert wurde nur Futterteig und Wasser, dazu wiederum der Darmsaft von ro nosemfreien Bienendarmen. 3) Neben Futterteig, Pollen, Darmsaft und Wasser wurden den Versuchsbienen noch 4o Stockbienen zugegeben, die ebenfalls wieder dem Baurahmen entstammten. Die drei Versuchsgruppen mit zusätzlich verabreichtem Darmsaft, der auf den Futterteig gestrichen wurde, sollten zeigen, ob durch den direkten Kontakt mit dem Darminhalt älterer Bienen eine Verstärkung des Flagellatenbefalles und dadurch eine zunehmende Schorfbildung erfolgen würde (Tab. r). Die Därme für die Zubereitung des Darmsaftes entstammten Bienen, deren Stockgenossen zu 64 p. 100 Pylorusschorfe besassen. Somit dürften auch in den zerriebenen Därmen Flagellaten vor- 
TABL,

Übersicht über das prozentuale Auftreten der Pylorusschorfe bei Arbeite-

Pourcentage d'apparition de l'escarre du pylore chez les ouv-

1. ohne Pollen

sans pollen.

2. mit Poller

avec polles

3. ohne Pollen, mit ' 40 älteren Stockbienen

sans pollen, avec 40 abeilles de ruches plus âgées.

4. mit Pollen, mit ' 0 älteren Stockbienen

avec pollen avec 40 abeilles de ruche plus âgées.....

5. mit Pollen, mit 40 älteren Stockbienen und Darmsaft älterer Stockbienen ..... avec pollen, avec 40 abeilles de ruche plus âgies et lo suc intestinal d'abeilles de ruche plus âgées

resamtzahl

Arbeitsbienen Nombre total d'abeilles

6. ohne Pollen, mit Darmsaft älterer Stockbienen ..... sans pollen, avec suc intestinal d'abeilles de ruche plus âgées

7. mit Pollen, mit Darmsaft âlterer Stockbienen

avec pollen, avec suc intest $i-$ nal d'abeilles de ruche plus ágées .............

8. Bienen eines Volkes am Flugloch gefangen

abeilles capturées au trou de vol d'une ruche........

9. Arbeitsbienen von Apis cerana

ouvrières d'Apis cerana ....

99

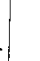

gresunde Bienen

Abeilles saines

\begin{tabular}{|c|c|c|c|c|}
\hline $\begin{array}{l}\text { Anzahl der } \\
\text { untersuchten }\end{array}$ & $\begin{array}{l}\text { kein Schorf } \\
\text { vorhanclen } \\
\end{array}$ & $\begin{array}{c}\text { Schorf } \\
\text { vorhanden } \\
\text { in }{ }^{\prime}\end{array}$ & $\begin{array}{c}\text { schwache } \\
\text { Verschorfung }\end{array}$ & $\begin{array}{c}\text { starke } \\
\text { Verschorfung }\end{array}$ \\
\hline
\end{tabular}

Bienen

Nombre

d'abeilles

in $\%$

Absence

d'escarres

$(\%)$

Présence

d'escarres

raible

in $\%$

testées

$(\%)$

$(\%)$

Forte escarri-

(\%) 
een der Honigbiene unter verschiedenen experimentellen Bedingungen

es d'abeilles dans différentes conditions expérimentales

\begin{tabular}{|c|c|c|c|c|c|c|c|c|}
\hline \multicolumn{5}{|c|}{$\begin{array}{l}\text { nosemakranke Bienen } \\
\text { Abeilles nosémosées }\end{array}$} & \multicolumn{4}{|c|}{$\begin{array}{l}\text { nosemakranke und gesunde Bienen } \\
\text { Abeilles nosémosées et Abeilles saines }\end{array}$} \\
\hline $\begin{array}{l}\text { nzahl der } \\
\text { tersuchten } \\
\text { Bienen } \\
\text { Nombre } \\
\text { d'abeilles } \\
\text { testées }\end{array}$ & $\begin{array}{l}\text { kein Schorf } \\
\text { vorhanden } \\
\text { in \% } \\
\text { Absence } \\
\text { d'escarres } \\
(\%)\end{array}$ & $\begin{array}{c}\text { Schorf } \\
\text { vorhanden } \\
\text { in \% } \\
\text { Présence } \\
\text { d'escarres } \\
(\%)\end{array}$ & $\begin{array}{c}\text { schwache } \\
\text { Verschorf- } \\
\text { ung in } \% \\
\text { Faible } \\
\text { escarrifi- } \\
\text { cation }(\%)\end{array}$ & $\begin{array}{c}\text { starke } \\
\text { Verschorf- } \\
\text { ung in } \% \\
\text { Forte } \\
\text { escarrif- } \\
\text { cation }(\%)\end{array}$ & $\mid \begin{array}{c}\text { kein Schorf } \\
\text { vorhanden } \\
\text { in \% } \\
\text { Absence } \\
\text { d'escarres } \\
(\%)\end{array}$ & \begin{tabular}{|c|} 
Schorf \\
Vorhanden \\
in \% \\
Présence \\
d'escarres \\
$(\%)$
\end{tabular} & $\begin{array}{c}\text { schwache } \\
\text { Verschorf- } \\
\text { ung in \% } \\
\text { Faible } \\
\text { escarrifi- } \\
\text { cation }(\%)\end{array}$ & $\begin{array}{c}\text { starke } \\
\text { Verschorf- } \\
\text { ung in } \% \\
\text { Forte } \\
\text { escarrifi- } \\
\text { cation }(\%)\end{array}$ \\
\hline 1 & 100 & 0 & 0 & 0 & 85 & 15 & 15 & 0 \\
\hline 6 & 66 & $33^{\prime}$ & 17 & 17 & 51 & 49 & 26 & 23 \\
\hline 59 & 14 & 86 & 86 & 0 & 16 & 81 & $8{ }^{\prime} t$ & 0 \\
\hline 35 & 46 & $5^{\prime}$ & 40 & $1{ }^{\prime}$ & $3 \dot{t}$ & 66 & 47 & 19 \\
\hline 30 & 17 & 83 & 63 & 20 & 26 & 74 & 56 & 18 \\
\hline 16 & 31 & 69 & 69 & 0 & $3{ }^{\prime}$ & 66 & 58 & 8 \\
\hline 2 & 50 & 50 & 0 & 50 & 16 & $84^{\prime}$ & 61 & 23 \\
\hline 46 & 30 & 70 & 5 & 65 & 36 & $6^{\prime}$ & 13 & 51 \\
\hline 0 & 0 & 0 & 0 & 0 & 100 & 0 & 0 & 0 \\
\hline
\end{tabular}


handen gewesen sein. Die den drei Versuchsgruppen zugegebenen Stockbienen waren Völkern entnommen, die nach vorangegangener Untersuchung als nosemafrei bezeichnet werden konnten. Die Jungbienen in den Versuchsgruppen ohne Pollennahrung wurden nach dem Schlüpfen sofort der Wabe entnommen. Sie hat ten dadurch keine Gelegenheit, eventuell aus der Wabe, von der sie stammten, Pollen zu fressen. Diesem Umstand musste Rechnung getragen werden, da die Bienen nach dem Schlüpfen sehr bald Pollen fressen (Schreiner und SEEgEr, i954). In den Versuchsgruppen mit Stockbienen wurden die Jungbienen gezeichnet. 24 'Tage nach dem Schlupf entnahm ich die noch überlebenden Bienen den Versuchskästchen und untersuchte sie auf etwaige vorhandene Pylorusschorfe. Bei 24 Tage alten Bienen ist zu erwarten, dass im Falle eines Flagellatenbefalles eine hinreichende Ausbildung der Pylorusschorfe hat vonstatten gehen können (GIAVARINI, I950). Vergleichsweise wurden neben den sieben Versuchsgruppen noch I2 I Flugbienen eines Bienenvolkes untersucht, und zwar entnommen am 25. V. 1966, desgleichen 105 Flugbienen eines Volkes von Apis cerana. Dieses Volk ist 1965 aus der Volksrepublik China importiert worden.

Trotz grosser Vorsichtsmassnahmen blieb es nicht aus, dass in einigen Versuchsgruppen Nosematose auftrat. Es konnten also zwangsläufig die Befunde bei gesunden Bienen denen nosemakranker Bienen gegenübergestellt werden.

Die unterschiedliche Grösse und Gestalt der Pylorusschorfe liess leicht eine Einteilung in starke und schwache Schorfe zu. Die schwache Verschorfung ist auf der äusseren Darmwand nur punktoder streifenförmig angedeutet, während die starken Schorfe auffällig dunkelbraun gefärbt sind und sich über mindestens $\mathrm{I} / 4$ der Darmwand in der Breite ausdehnen. Mitunter nehmen sie den halben oder gar den ganzen Darmumfang am Pylorus ein. In einigen Fällen wurden die Schorfe auf Flagellatenbesat\% untersucht. Jedesmal konnten Flagellaten festgestellt werden.

\section{UNTERSUCHUNGSERGEBNISSE UND DISKUSSION}

Die wesentlichen Ergebnisse der vorliegenden Untersuchungen sind in Tabelle I zusammengefasst. Darin kommt zum Ausdruck, dass in den drei Versuchsgruppen ohne Pollennahrung starke Pylorusschorfe $(+)$ überhaupt nicht (zweimal) bzw. einmal nur zu 8 p. Ioo beobachtet werden konnten. Dieses Ergebnis ändert sich nur unwesentlich, wenn man die nosemakranken und die nicht nosemakranken Gruppen getrennt betrachtet. Interessant ist in diesem Zusammenhang, dass KLUGE (I963) bei Verfütterung von 6 Monate altem Bienenbrot an Stelle von frischem Pollen keine Darmflagellaten gefunden hat. Ubereinstimmend mit den vorliegenden Ergebnissen ist der Mangel stärkerer Flagellatenansammlungen bzw. Schorfbildungen bei fehlender Pollennahrung. Das gänzliche Fehlen von Flagellaten bei der Verfütterung von altem Bienenbrot lässt sich vielleicht mit der Zusammensetzung dieser Nahrung in Verbindung bringen. In den vier Versuchsgruppen mit Pollennahrung hingegen sind starke Verschorfungen relativ häufig ( $18-23$ p. roo). Bei den am Flugloch eines Volkes gefangenen Bienen beträgt die Anzahl der Bienen mit starken Verschorfungen sogar 5I p. I0o. Wiederum ist kein merklicher Unterschied zwischen gesunden und nosemakranken Bienen zu erkennen. Die $50 \mathrm{p}$. Ioo ( +) bei den nosemakranken Bienen der Versuchsgruppe 7 besitzen keinen Aussagewert, da insgesamt nur zwei nosemakranke Bienen in dieser Gruppe vorhanden waren.

Weiterhin ist zu bemerken, dass der geringe Prozentsatz der Schorfbildung in der I. Versuchsgruppe (I5 p. IOO) in keiner der anderen Gruppen wiederkehrt oder gar unterboten würde. In Gruppe 3 u. 6 (Eirnährung ohne Pollen) ist die geringfügige Verschorfung sogar recht beträchtlich. Das mag damit zusammenhängen, dass in der einen Gruppe ältere Bienen mit den Versuchsbienen zusammen eingesperrt waren und in der anderen Gruppe Darmsaft verfüttert worden war. In beiden Fällen ist die UUbertragung und Entwicklung der Flagellaten trotz mangelnder Pollener- 
nährung der Wirtstiere vermutlich durch eine beträchtliche Infektion ermöglicht worden. Aber auch in den auf diese beiden unterschiedlichen Arten infizierten Versuchsgruppen ist bei zusätzlicher Pollennahrung die starke Verschorfung häufiger als bei den übrigen Gruppen. Das wesentliche Ergebnis dieser Untersuchung, das sich einmal aus den mit Pollenzufütterung, zum anderen bei den ohne Pollen ernährten Gruppen ergeben hat, wird durch die hinzukommende Infektion durch ältere Bienen oder durch Verfütterung von Darmsaft älterer Bienen nur etwas modifiziert, nicht aber grundsätzlich verändert. Die Verfütterung von Darmsaft an die Jungbienen scheint zu keinem stärkeren Flagellatenbesatz mit nachfolgender Verschorfung zu führen, als wenn die frisch geschlüpften Bienen allein durch ältere Stockbienen infiziert werden. Auf die Zunahme des Flagellatenbefalles bei Jungbienen, die mit älteren Bienen in Berührung gekommen sind, macht schon HIschIER (I962) aufmerksam. Die Übertragung der Flagellaten von den älteren Stockbienen auf die Jungbienen liesse sich damit erklären, dass die älteren Bienen in den Versuchskästchen hin und wieder Kot absetzen. Vielleicht spielt aber auch die Trophallaxis dabei eine Rolle. Nach BURrI (I944, I947, zit. nach Trommer, I965) sind frisch geschliipfte Bienen frei von Mikroorganismen. Die Flagellaten werden später offensichtlich per os aufgenommen, dabei diirfte die Trophallaxis normalerweise von Bedeutung sein, was nach KLUGE (Ig63) auch für die Aufnahme von Bakterien zutrifft.

Da die sessilen Pylorusflagellaten saprophytisch leben (LoTMAR, I946) und abgesehen von Veränderungen der Darmwand an den Anheftungsstellen (B̈̈HRMANr, noch unveröffentlicht) keine weiteren Schädigungen im Wirtsorganismus verursachen, üben sie anscheinend auch keinen Einfluss auf die Lebenslänge der Bienen aus. Die unterschiedliche Lebenslänge der einzelnen Versuchsgruppen ist in erster Linie wohl auf die Zusammensetzung der Nahrung zurückzuführen. In den Kästchen ohne Pollenfütterung lebten nach 24 Tagen noch 7 I (3), 53 (I) und $3^{8}$ (6) von anfangs I2O vorhandenen Bienen. Dieses Ergebnis steht im Einklang mit den Ermittlungen von BeUtLER und OPFINGER (I949), HAydak (I937) und MaURizio (I946) über die hohe Sterblichkeit beim Fehlen der Pollennahrung bzw. die lebensverlängernde Wirkung des Pollens.

Die Form der Pylorusschorfe kann vor allem bei starker Verschorfung recht unterschiedlich sein. Die Schorfe können auf der Dorsalseite des Pylorus begrenzte rundliche oder auch eckige Flecke darstellen; sie können sich aber auch bandförmig quer über den Pylorus ziehen, wobei sie im Gegensatz zu den Mitteilungen von GIAVARINI (I950), Hischier (I962) und LotMAR (I946) nicht auf die Dorsalseite des Darmes beschränkt bleiben müssen. Das trifft sowohl für die Versuchsbienen als auch für Bienen aus dem Volke zu. Bei 24 der untersuchten Bienen umschliessen die Schorfe den gesamten Darmquerschnitt ringförmig. Bezeichnenderweise treten solche starken Verschorfungen nur bei Bienen auf, die mit Pollen ernährt worden sind. Einmal konnte ein Schorf rechts seitlich am Pylorus ermittelt werden. Die Schorfe, die den Darm ringförmig umschliessen, zeigen häufig längs parallel liegende Versteifungen. Diese sehr dunkel gefärbten Teile wechseln mit helleren Schorfpartien ab. Vielfach ist die Darmwand an den Stellen starker Verschorfung konkav eingebuchtet. 


\section{RÉSUMÉ}

\section{SUR LA PRÉSENCE D'ESCARRES PYLORIQUES \\ CHEZ LES OUVRIÈRES D'ABEILLES SAINES OU NOSÉMOSÉES EN CONDITIONS EXPÉRIMENTALES DIVERSES}

Plusieurs auteurs (Lotmar, Hischier, Giavarini) ont déjà signalé chez les abeilles, la présence de flagellés, qui sous leur forme sessile, à leur point de fixation dans le proctodeum, au niveau du pylore, provoquent des escarres de plus ou moins grande importance.

L'auteur en tenant compte de ces travaux s'est posé la question de savoir quel facteur pouvait être responsable de l'apparition de flagellés et par conséquent d'escarres du pylore.

C'est pourquoi, il a examiné l'influence exercée par une alimentation à base de pollen et par le contact entre jeunes et vieilles ouvrières sur la formation des escarres.

Des groupes de r zo abeilles (Apis mellifica) saines ou nosémosées ont été maintenues dès leur naissance jusqu'à l'âge de 24 jours, sous diverses conditions expérimentales. Les ouvrières ont reçu :

I. Une alimentation à base de candi (sucre en poudre et miel) avec ou sans addition de pollen frais, pris dans un cadre ;

2. Une alimentation à base de candi avec ou sans pollen, l'auteur ajoutant aux i 20 ouvrières expérimentales 40 abeilles de ruches âgées d'au moins 8 jours ;

3. Une alimentation avec ou sans pollen; sur le candi était répandu le suc de ro intestins postérieurs broyés en provenance d'abeilles exemptes de nosémose ;

4. Une alimentation à base de pollen et de candi additionné de suc intestinal, en présence de 40 abeilles de ruche plus âgées.

L'auteur a constaté, chez les groupes d'ouvrières alimentées de pollen, la présence relativement fréquente d'escarres. Celles-ci sont importantes, colorées en brun foncé et ell ss s'étendent en largeur sur au moins le quart de la paroi intectinale et quelquefois sur tout le pourtour de l'intestin au niveau du pylore.

En l'absence de pollen, il y a moins d'escarres que lorsque les abeilles reçoivent du pollen. Ces escarres sont considérées comme faibles. La différence est significative $(\mathbf{P}=0,0 \mathrm{r})$.

Chez les groupes d'ouvrières accompagnées d'abeilles de ruche ou nourries avec addition de suc intestinal d'abeilles plus âgées, la présence fréquente d'escarres de faible importance est notée, même en l'absence d'une alimentation à base de pollen. En présence de pollen, les escarres sont plus étendues et leur présence est plus fréquente que dans les autres groupes.

L'auteur n'a pas constaté de différence dans le nombre et l'importance des escarres du pylore entre les abeilles saines et les abeilles nosémosées. L'auteur ajoute (communication non publiée) qu'étant donné que les flagellés sous la forme fixée vivent en saprophytes, à part les modifications de la paroi intestinale qu'ils peuvent provoquer à l'endroit de leur fixation, ils n'exercent aucune influence sur la longévité des abeilles.

\section{SUMMARY}

\section{ON THE OCCURRENCE OF PYLORIC SCABS IN HEALTIY AND NOSEMA-AFFECTED} HONEYBEE WORKERS UNDER VARIOL'S EXPERIMENTAL CONDITIONS

Several authors (Lotmar, Hischier, Giavarini) have described the presence of flagellates in bees, which in their sessile form cause scabs of varying degrees at their point of fixation in the proctodaeum at the level of the pylorus.

To discover what was responsible for the appearance of flagellates and the consequent scabs on the pylorus, an examination was made of the effect of feeding with a pollen base and of the contact between young and old workers.

Groups of $\mathrm{I} 20$ healthy or nosema-affected bees (Apis mellifica) were kept from birth up to the age of 24 days under various experimental conditions. The workers received : a frame;

I. food with a candy base (powdered sugar and honey) with or without fresh pollen, taken from

2. food with a candy base with or without pollen, 40 bees from the hive aged at least 8 days being added to the I 20 experimertal workers;

3. food with or without pollen, the juice of Io ground posterior intestines from nosema-exempt bees being scattered on the candy; 
4. food with a pollen and candy base with added intestinal juice, in the presence of 40 older bees from the hive.

Scabs were found to be relatively frequent in the pollen-fed groups of workers. The scabs were large and dark brown, and covered at least a quarter of the intestinal wall and sometimes all the periphery of the intestines at the level of the pylorus.

There were fewer scabs when no pollen was given and these scabs were small. The difference is significant $(\mathrm{P}=0.0 \mathrm{I})$.

Small scabs were frequently present in the groups of workers accompanied by bees from the hive or fed with an addition of intestinal juice from older bees, even with no pollen-based food. When pollen was present, the scabs were more widespread and more frequent than in the other groups.

No difference was found between the healthy and the nosema-affected bees in the number and size of the pyloric scabs. In the fixed form the flagellates live as saprophytes. and although they modify the intestinal wall where they are fixed, they have no effect on the bees' lifetime.

\section{ZUSAMMENFASSUNG}

I. In verschiedenen Versuchsgruppen wurden je I20 frisch geschlüpfte Arbeitsbienen mit und ohne Pollenzufütterung zu Honig-Staubzucker-Teig gehalten. In einigen Versuchsgruppen wurden den Jungbienen je 4o ältere Stockbienen beigegeben, auch wurde Darmsaft älterer Bienen verfüttert. Nach 24 Tagen wurden die Versuchsbienen auf das Vorhandensein und die Stärke der Pylorusschorfe untersucht.

2. Bei fehlender Pollennahrung sind weniger Pylorusschorfe vorhanden als wenn den Bienen auch Pollen verabreicht wird. Der Unterschied ist signifikant $(\mathrm{P}=0, \mathrm{oI})$.

3. Leben die Versuchsbienen mit älteren Stockbienen zusammen, oder/und wird zusätzlich Darmsaft älterer Bienen verfüttert, erhöht sich auch bei fehlender Pollennahrung der Prozentsatz der Bienen mit schwachen Pylorusschorfen. Starke Verschorfungen sind aber auch dann kaum oder gar nicht zu beobachten.

4) Unterschiede in der Zahl und der Stärke der Pylorusschorfe bei gesunden und nosemakranken Bienen sind nicht vorhanden.

\section{LITERATURVERZEICHNIS}

BÄHRMANN R. (noch unveröffentlicht) : Vergleichend-histologische Untersuchungen am Enddarm und den Malpighischen Gefässen gesunder und nosemakranker Honigbienen unler besonderer Berïcksichtigung der Darmfiagellaten Leptomonas apis LOTMAR.

Beutler R., E. Opfinger, I949. Pollenernährung und Nosemabefall der Honigbiene (Apis mellifica L.). Z. vergl. Physiol., 32, $383-421$.

Fyg W., ig54. Über das Vorkommen von Flagellaten in Rectum der Honigbiene (Apis mellifica L.). Mitt. Schweiz. Entomol. Ges., 27, 423-428.

Giavarini I., i950. Sui flaggellati dell' intestino tenue dell' Ape Domestica. Suppl. Boll. Zool., 17,603-608. HAYDAK M., I937. The influence of the pure carbohydrate diet on newly emerged honeybees. Ann. Entomol. Soc. Amer., 30, 258-262.

Hischier J., I962. Uniersuchungen über Flagellalen im Darm der Honigbiene. Diss. Bern.

Kudge R., 1963. Untersuchungen über die Darmflora der Ionigbiene Apis mellifica L. Z. Bienenforsch., 6, I4I-169.

Lotmar R., 1946. Über Flagellaten und Bakterien im Dünndarm der Honigbiene. Beihefte Schweiz. Bienenztg, 2 (I4).

Maurizio A., I946. Beobachtungen über die Lebensdauer und den Futterverbrauch gefangen gehaltener Bienen. Beihelte Schweiz. Bienenztg, 2 (1 3 ).

NöILER W., I931. Die nächsten Verwandten der Blutflagellaten und ihre Beziehungen zu den blutbewohnenden Formen. In : Prow

Schreiner Th., H. Seeger, i954. Volumenmessungen am Mitteldarm der Honigbiene. Z. Nalurforsch. $9 b: 69-76$.

TROMmer F., I965. Qualilative und quantitative Untersuchungen über die aerobe und anaerobe Darmflora des Mitteldarmes der gesunden und nosemakranken Honigbiene. Diss. Leiprig. 